\title{
Removal of Gaussian noise on the image edges using the Prewitt operator and threshold function technical
}

\author{
H. A. Alshamarti \\ Department of Physics, College of Science,University of Kufa, Iraq
}

\begin{abstract}
Image edge detection algorithm is applied on images to remove Gaussian noise that is present in the image during capturing or transmission using a method which combines Prewitt operator and threshold function technique to do edge detection on the image. This method is better than a method which combines Prewitt operator and mean filtering. In this paper, firstly use mean filtering to remove initially Gaussian noise, then use Prewitt operator to do edge detection on the image, and finally applied a threshold function technique with Prewitt operator.
\end{abstract}

Keywords: Gaussian noise, Prewitt operator, edge detection, threshold function

\section{Introduction}

The image edge contains some of the useful information in the image. It uses to measures the size of objects in the image, isolate particular objects from their background, and recognize objects. There are a large number of edge-finding algorithms in existence [1]. The image processing using computers has created suitable images for people to observe, identify, recognize, and understand images. Edge detection is one of research keys in image processing [2]. Edge detection is one of the fundamental operations in image processing and computer vision. It is defined as the process of locating the boundaries of objects or textures showed on the image. The positions of these boundaries are defined critical in the process of image enhancement, recognition, restoration, and compression $[3,4]$.

\section{Gaussian Noise And Mean Filter}

The camera and scanner devices capture the image and these devices sometimes add extraneous noise to the image. This noise should be removed from the image for other image processing operations to return valuable results. Noise on the image is the result of errors in the image acquisition process that result in pixel values that do not reflect the true intensities of real image. The presence of noise gives the image a grainy, rough, mottled, and snowy appearance. The image noise magnitude can be varied from gradual specks to optical and radio astronomical images that are completely noise. The various types of noises on the image are presented as Gaussian, salt and pepper, speckle noises [5].

The probability density function (PDF) of Gaussian noise is equal to that of the normal distribution (Gaussian distribution). It is used as additive white noise to give additive white Gaussian noise (AWGN), where all the image pixels deviate from their original values following Gaussian curve for each image pixel with intensity value $\mathrm{f}_{\mathrm{ij}}\left(1 \leq \mathrm{i} \leq \mathrm{m}, 1 \leq \mathrm{j} \leq \mathrm{n}\right.$ for an $\mathrm{m} * \mathrm{n}$ image), the corresponding pixel of the noisy image $\mathrm{g}_{\mathrm{ij}}$ is $[6,7]$

$$
\mathrm{g}_{\mathrm{i}, \mathrm{j}}=\mathrm{f}_{\mathrm{i}, \mathrm{j}}+\mathrm{n}_{\mathrm{i}, \mathrm{j}}
$$

where each noise value (n) is drawn from a zero-mean Gaussian distribution [8].

Mean filter is broadly used in reducing Gaussian noise and smoothing the images as shown equation (2) [9]. Average filter or median filter is simple, instinctive, and easy to understand. It reduces the amount of intensity variation between one pixel and the next. Each pixel value on the image is replaced with the average value of its neighbors including that pixel [10]. The mean filter is a linear filter which uses a mask over pixel in the signal. Each of the components of the pixels which fall under the mask are averaged to form a single. The mean filter is

$$
\text { mean filter }\left(I_{1} \ldots \ldots \ldots \ldots I_{N}\right)=\frac{1}{N} \sum_{i=1}^{N} I_{i}
$$

where $\left(I_{1} \ldots \ldots \ldots I_{N}\right)$ is the image pixel range [11].

\section{Edge Detection Principle}

The edge mostly exists between objects and background, objects and primitives. It contains rich information, step property, shape, which is able to describe the target object. Edge detection operations are 
based on the idea that edge information on an image is found by looking at the relationship a pixel has with its neighbors. If a pixel has gray level values similar to those around it, there is probably not an edge at that point. However, if a pixel has neighbors with varying gray levels, it may represent an edge point. The edge is defined by a discontinuity in gray-level values [1]. There are two types of edge detection. The first derivative-based edge detection operator to detect image edges by computing the image gradient values such as Roberts, Sobel, and Prewitt operators. The second derivative-based edge detection operator is such as LOG, Canny operators [9].

\subsection{Prewitt Operator}

The Prewitt operator masks edges in both the horizontal and vertical directions and then combines this information into a single metric. The masks are as follows

\begin{tabular}{cccccc}
-1 & -1 & -1 & -1 & 0 & 1 \\
0 & 0 & 0 & -1 & 0 & 1 \\
1 & 1 & 1 & -1 & 0 & 1 \\
\multicolumn{2}{l}{ Row Mask $\left(\mathrm{P}_{1}\right)$} & & Column Mask $\left(\mathrm{P}_{2}\right)$ &
\end{tabular}

These masks are convolved with the image. The edge magnitude and edge direction are defined by

$$
\begin{aligned}
& \text { Edge Magnitude }=\sqrt{\mathrm{P}_{1}^{2}+\mathrm{P}_{2}{ }^{2}} \\
& \text { Edge Direction }=\tan ^{-1} \frac{P_{1}}{P_{2}}
\end{aligned}
$$

\subsection{Threshold Function Technique}

The Image threshold technique is a common task in computer vision and graphic applications. The goal of image threshold technique is classify pixels as either "dark" or "light". Adaptive of threshold is a form of threshold that takes into account spatial variations in illumination [12]. Let $\mathrm{N}$ be natural numbers, $(\mathrm{x}, \mathrm{y})$ be the spatial coordinates of a digitized image, and $\mathrm{G}(0,1 \ldots \mathrm{I}-1)$ be positive integers. The brightness (gray level) of a pixel with coordinates $(\mathrm{x}, \mathrm{y})$ is denoted as $\mathrm{f}(\mathrm{x}, \mathrm{y})$. Consider a original image in which $g(x, y) \in[0,255]$ be the intensity of a pixel at location $(x, y)$. A threshold $t(x, y)$ for each pixel is computed by

$$
I(x, y)=\left\{\begin{array}{r}
\mathbf{0} \text { if } g(x, y) \leq t(x, y) \\
\mathbf{f}(\mathbf{x}, \mathbf{y}) \text { if } g(x, y) \geq t(x, y)
\end{array}\right.
$$

Method and algorithms are:

\section{Method And Algorithms}

a. Conversion of image to gray level.

b. Gaussian noise addition for the image $(\sigma=0.005: 0.005: 0.05)$.

c. Application of mean filter on the image.

d. Using Prewitt operator (input image).

e. Using the threshold function technique (output image).

\section{Analysis And Experimental Results}

Figure1 shows original image used in this study. Gaussian noise is added to the image (Figure 2). Figure 3 shows application of the mean filter to remove the primary noisy images. Figure 4 shows using the Prewitt operator of edge detection.

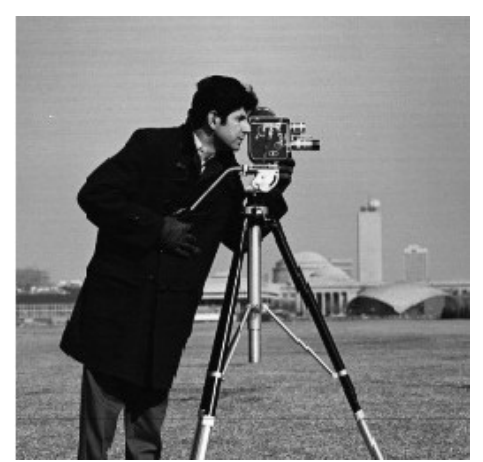

Figure 1. Original image 
ARAR

intar

AnRA

Arrar 


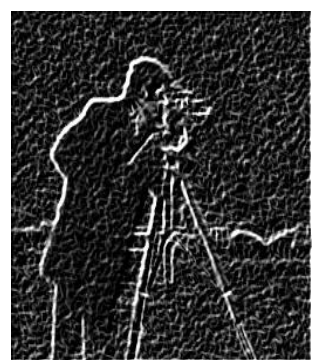

(6)

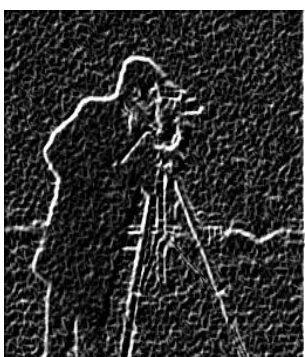

(7)

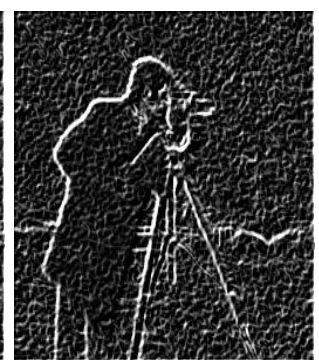

(8)

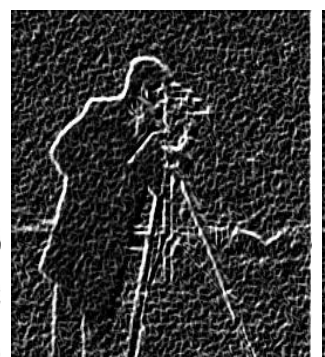

(9)

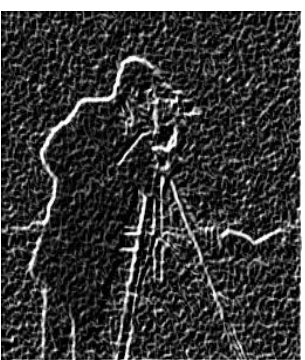

(10)

Figure 4. Mean filter and Prewitt operator, window $(3 * 3)$ for (Figure 3 )

The methods which combine mean de-noising and Prewitt operator are commonly used to find the edge. However, these methods cannot remove Gaussian noise very well. This paper proposes Prewitt operator of edge detection based on threshold function technique. It can be removed the noise effectively and can detect the edge detail very well. The results are showed in Figure 5.

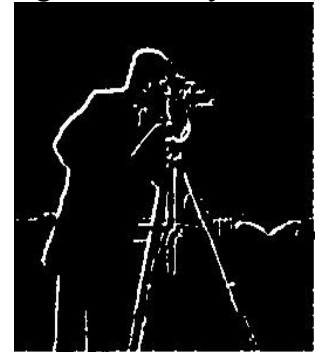

(1)

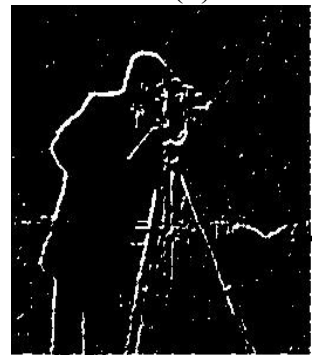

(6)

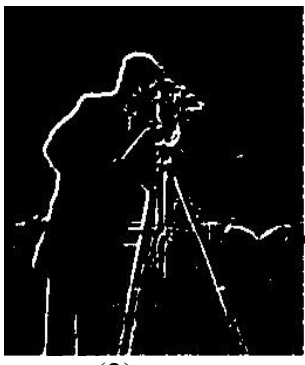

(2)

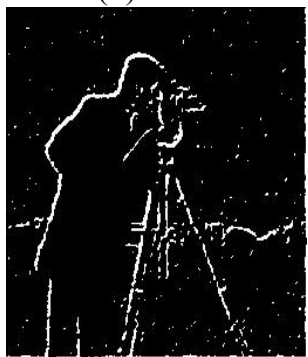

(7)

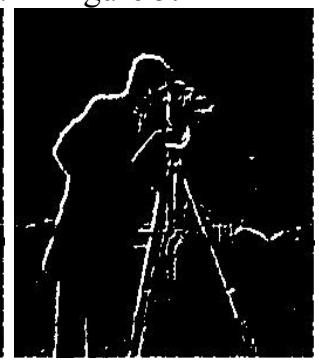

(3)

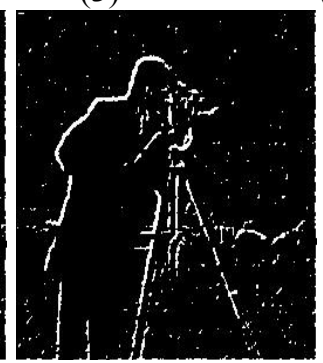

(8)

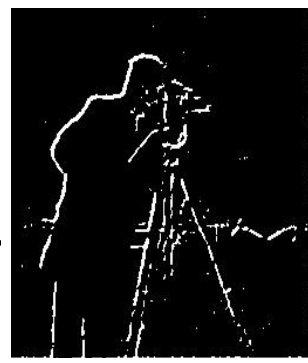

(4)

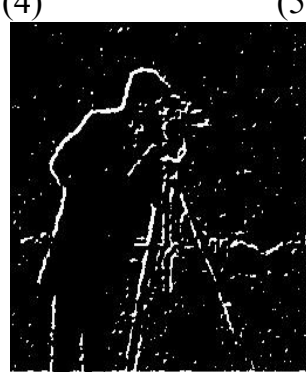

(9)

(10)

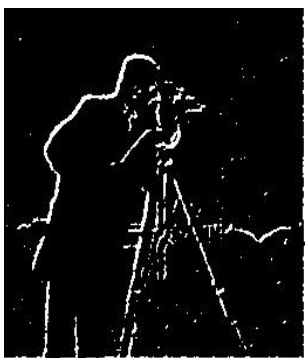

(5)

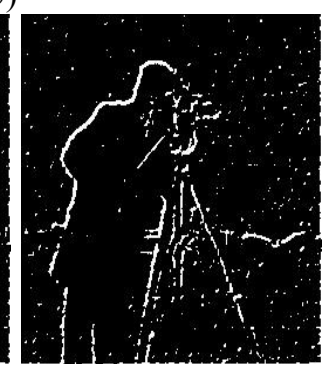

Figure 5. Prewitt operator based on threshold function technique (threshold value $=160$ )

\section{Conclusions}

Prewitt operator methods with mean filter failed to perform adequately to remove Gaussian noise that have different amount (0.005:0.005:0.05). In this work, the Prewitt operator with threshold technique (threshold value $=160)$ is used very well for Gaussian noise removal on image.

\section{Acknowledgments}

The author is grateful to Dr. Basim A. Almayahi, Department of Environment, College of Science, University of Kufa for assisting me throughout conducting the present research. Financial support was provided by the College of Science, University of Kufa.

\section{Reference}

[1] R. Gonzalez, R. E. Woods. Digital Image Processing. Addison-Wesley, $2^{\text {nd }}$ Ed., 2002.

[2] W. Gao, L. Yang, X. Zhang, H. Liu, 2010. An Improved Sobel Edge Detection. 978-1-4244-5540-9/10. IEEE.

[3] D. Ziou, S. Tabbone, 1998. Edge detection techniques - an overview, International Journal of Pattern Recognition and Image Analysis, 8, 537-559.

[4] M. Qasim, W. L. Woon, Z. Aung, V. Khadkikar, 2013. Intelligent Edge Detector Based on Multiple Edge Maps. 978-1-4673-51577/13. IEEE.

[5] S. Dangeti, 2003. De-noising Techniques: a Comparison. M.S. Thesis. Louisiana State University.

[6] S. Kumar, P. Kumar, M. Gupta, A. K. Nagawat, 2010. Performance Comparison of Median and Wiener Filter in Image De-noising. International Journal of Computer Application, 12, 4.

[7] S. Zhong, V.Cherkassky, 2000. Image de-noising using wavelet thresholding and Model selection. IEEE. 
[8] G. Ilango, R. Marudhachalam, 2011. New hybrid filtering techniques for removal of gaussian noise from medical images. ARPN Journal of Engineering and Applied Sciences, 6, 2.

[9] L. Bin, M. S. Yeganeh, 2012. Comparison for image edge detection algorithms. IOSR Journal of Computer Engineering (IOSRJCE), 2, 01-04.

[10] J. C. Church, Y. Chen, S.V. Rice, 2008. A spatial median filter for noise removal in digital images. IEEE, 618-623.

[11] G. Padmavathi, P. Subashini, M. M. Kumar, S. K. Thakur, 2009. Performance analysis of Non Linear Filtering Algorithms for underwater images. (IJCSIS) International Journal of Computer Science and Information Security, 6, 2.

[12] F. Shafaita, D. Keysersa, T. M. Breuel. Efficient implementation of local adaptive thresholding techniques using integral images. German Research Center for Artificial Intelligence (DFKI) GmbH D-67663 Kaiserslautern, Germany. 